\title{
The role of prenatal steroids at 34-36 weeks of gestation
}

\author{
Gordon CS Smith, ${ }^{1}$ David Rowitch, ${ }^{2}$ Ben WJ Mol ${ }^{3}$
}

A recent multicentre, double-blind, randomised, placebo-controlled trial has demonstrated that administration of betamethasone to women with threatened preterm delivery at 34-36 weeks of gestational age reduces the risk of neonatal respiratory morbidity. There is, however, no long-term outcome data on the children, and we believe that it is biologically plausible that this treatment may cause long-term harm through effects on the infant's brain. Given this, we argue that steroids should not be used in the context of late preterm delivery until evidence of long-term safety is available. This example illustrates some strengths and weaknesses of using 'levels of evidence' to grade the empirical support for making clinical decisions.

One of the major advances in perinatal medicine in the last 30 years has been the administration of synthetic glucocorticoids to mothers who are likely to deliver at extreme preterm gestational ages, and this intervention clearly reduces perinatal mortality and severe morbidity. ${ }^{1}$ A recent multicentre, double-blind, randomised controlled trial (RCT) compared the effects of betamethasone versus placebo among women presenting between 34 and 36 weeks of gestational age with a high probability of delivery. ${ }^{2}$ The primary outcome was need for respiratory support 3 days after delivery. The rate was $11.6 \%$ in steroid-treated children and 14.4\% with placebo, yielding a number needed to treat of 35 (95\% CI 19 to 259). In addressing the question of whether this trial justifies immediate incorporation of glucocorticoids into the management of threatened delivery at 34-36 weeks, we need to consider both the science of

\footnotetext{
${ }^{1}$ Department of Obstetrics and Gynaecology, Cambridge University, Cambridge, UK

${ }^{2}$ Department of Paediatrics, Wellcome Trust-MRC Stem Cell Institute, University of Cambridge, Cambridge, UK ${ }^{3}$ Department of Obstetrics and Gynaecology, The Robinson Research Institute, School of Medicine, University of Adelaide, Adelaide, Australia
}

Correspondence to Professor Gordon CS Smith Department of Obstetrics and Gynaecology, University of Cambridge, NIHR Cambridge Comprehensive Biomedical Research Centre, Box 223, The Rosie Hospital, Cambridge CB2 2SW, UK; gcss2@cam.ac.uk glucocorticoids in pregnancy and the science of clinical trials.

Physiologically, the fetus prepares for birth near term by an increased production of cortisol from the fetal adrenal. The glucocorticoids employed therapeutically to accelerate fetal lung maturation are betamethasone and dexamethasone. The choice is purposeful as both are resistant to $11 \beta$-hydroxysteroid dehydrogenase-2 (11 $\beta$-HSD-2), ${ }^{3}$ an enzyme that is highly expressed in the placenta and that limits transplacental passage of maternal cortisol by converting it to inactive metabolites. Consequently, steroids that are metabolised by $11 \beta$-HSD-2 (such as cortisol, hydrocortisone and prednisolone) cannot cross the placenta, whereas $11 \beta$-HSD-2-resistant steroids can. Importantly, the fetal brain has high levels of expression of $11 \beta-H S D-2$, especially in areas that undergo rapid growth in the third trimester (eg, the cerebellum). ${ }^{4}$ As a result, the brain is relatively protected from the effects of the physiological rise in endogenous cortisol in late gestation. ${ }^{5}$ It follows, however, that maternally administered betamethasone or dexamethasone near term will both cross the placenta and activate brain glucocorticoid receptors due to the resistance of these drugs to metabolism by $11 \beta-H S D-2$. Hence, betamethasone and dexamethasone will cause unphysiological activation of glucocorticoid receptors in the fetal brain near term.

Should we be concerned? There are a number of observations that suggest we should. First, it has been shown that $11 \beta$-HSD-2-resistant glucocorticoids inhibited normal cerebellar growth when administered to neonatal mice ${ }^{6}$ and had profound and long-lasting epigenetic effects on the brain of fetal guinea pigs following maternal administration in late gestation. ${ }^{7}$ Second, the one study that previously examined long-term outcomes following betamethasone in late pregnancy (given prior to prelabour caesarean section at 37 or 38 weeks of gestational age) demonstrated that glucocorticoid-exposed children were twice as likely to be identified as being in the lowest achievement group at school compared with the control group (17.7\% vs $8.5 \%$, relative risk 2.1 ; $95 \%$ CI 1.1 to
3.7). ${ }^{8}$ Interestingly, the same trial demonstrated no long-term benefit of steroids. Part of the motivation for the original trial had been that prevention of short-term respiratory morbidity around the time of birth may reduce the risk of respiratory disease in later childhood. However, the long-term follow-up study demonstrated no such benefits. Hence, the justification for glucocorticoids prior to term prelabour caesarean section is wholly based on reducing the risk of short-term complications.

A number of studies have compared longer term follow-up (in children ${ }^{1}$ and adults $^{9}$ ) in those exposed to antenatal glucocorticoids and placebo controls from the original RCTs of glucocorticoids in women presenting with threatened preterm birth. However, these studies generally assessed the effects of steroid exposure at earlier gestational ages. Overall, these results are reassuring and support the use of steroids in the management of preterm birth prior to 34 weeks. ${ }^{1}$ However, the apparent longterm safety of steroids following exposure at more extreme degrees of prematurity does not confirm their safety at later gestational ages. Potential direct harmful effects of steroids on the brain could be masked by the generally protective effect of improved neonatal cardiorespiratory function and reduced risks of specific acute perinatal complications, such as intraventricular haemorrhage. Moreover, activation of the glucocorticoid receptor by steroids is regulated by multiple aspects of the cellular and physiological context, termed 'context-driven plasticity'. ${ }^{10}$ It is possible-indeed likely-that the effect of a given concentration of a given steroid may differ at different gestational ages due to the multiple factors that change with advancing gestational age and the complex regulation of glucocorticoid receptor-mediated responses.

In the context of extreme preterm birth (eg, 24-28 weeks' gestational age), the balance of risks and benefits favours the use of glucocorticoids, as they have clearly been shown to prevent death and severe adverse outcome in the short term and long term. ${ }^{1}$ However, in our view, the case has not been established that the balance of risks and benefits favours their use after 33 weeks of gestational age. We know that the use of steroids in women with threatened delivery at 34-36 weeks means that 35 children need to be treated to prevent one child requiring respiratory support 3 days after delivery. What we do not know is the long-term effects of fetal exposure to these drugs at this gestational age, 
that is, whether the prevalence or severity of long-term adverse effects-if there are any-outweigh the known benefit.

This example also illustrates a series of key points about RCTs in perinatal medicine: (1) there are multiple outcomes that may be affected by an intervention, (2) the effect of an intervention might differ proportionally and directionally for different outcomes and (3) the level of evidence around different effects may differ. In the case of late preterm glucocorticoids, their beneficial short-term effect is supported by level 1 evidence. In contrast, the potential for adverse effects on the fetal brain is largely based on level 5 evidence, that is, expert opinion and basic research.

A relative lack of evidence on rare and long-term outcomes is a general feature of trials and reflects some practical realities: trials are expensive and the larger they are and the longer the follow-up, the more expensive the trial. Consequently, trials are biased to be powered for outcomes that are common and occur in the short term. In contexts such as extreme preterm birth and cancer, the clinically most important outcomes, such as death, may be common and occur in the short term. However, in many other contexts, including late preterm delivery, common and short-term complications are generally not severe, and the most important outcomes may be rare and/or long term, including harm done by the intervention. A danger of RCTs in obstetrics is that trials may not be funded or powered to detect long-term adverse outcomes in children.

Ideally, the absence of level 1 evidence for all of the major outcomes of interest would not be a problem. The lack of information on long-term outcomes, or underpowered analyses leading to wide CIs, would be interpreted as indicating that there is no accurate information about whether these outcomes are affected by the intervention, and the absence of evidence would be a cause for concern. However, it has been suggested that the six most dangerous words in evidence-based medicine are: 'there is no evidence to demonstrate'. ${ }^{11}$ If the 'absence of evidence' is conflated with 'evidence of absence', we will be misled. The correct summary of using glucocorticoids at 34-36 weeks is that there is evidence of short-term benefit but an absence of evidence of long-term safety. However, there are those who might state that there is evidence of short-term benefit and no evidence to demonstrate long-term harm. The different wording yields a profound difference in inference.

Randomised trials have had a hugely positive effect on the practice of obstetrics and paediatrics. The strength of trials is that this study design has the highest internal validity, all other things being equal. However crucially, we cannot assume no effect for questions where there are limited or no data. Therefore, when we assess the evidence base we must consider all the issues. The problem is not trials. The problem is a simplistic approach to a complex question. Labelling evidence on the basis of study design alone, without reference to statistical power, particular attention to adequate length of follow-up, harm, external validity or biological plausibility is treating a complex question in a simplistic manner. We ignore complexity at our peril or, rather, at the peril of our patients.

Contributors GCS had the original idea. All authors contributed to the manuscript, and all authors saw and approved the final version.

Competing interests GCSS receives/has received research support from GE, Roche and GSK. GCSS has been paid to attend advisory boards by GSK and Roche and to consult for GSK. GCS has received support to attend a scientific meeting from Chiesi. GCSS is named inventor in a patent submitted by GSK for a novel application of an existing GSK compound for the prevention of preterm birth (PCT/EP2014/062602). GCSS is a member of a GSK Data Safety Monitoring Committee for a trial of RSV vaccination in pregnancy and infancy. BWJM has been paid to attend advisory boards for ObsEva, Geneva, and has presented at sponsored scientific meetings.

Provenance and peer review Commissioned; externally peer reviewed.

(c) Article author(s) (or their employer(s) unless otherwise stated in the text of the article) 2017. All rights reserved. No commercial use is permitted unless otherwise expressly granted.

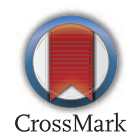

To cite Smith GCS, Rowitch D, Mol BWJ. Arch Dis Child Fetal Neonatal Ed 2017;102:F284-F285.

Received 1 March 2017

Accepted 12 March 2017

Published Online First 4 April 2017

Arch Dis Child Fetal Neonatal Ed 2017;102:F284-F285. doi:10.1136/archdischild-2016-312333

\section{REFERENCES}

1 Roberts D, Dalziel S. Antenatal corticosteroids for accelerating fetal lung maturation for women at risk of preterm birth. Cochrane Database Syst Rev 2006;(3):CD004454.

2 Gyamfi-Bannerman C, Thom EA, Blackwell SC, et al; NICHD Maternal-Fetal Medicine Units Network. Antenatal betamethasone for women at risk for late preterm delivery. N Engl J Med 2016;374:1311-20.

3 Aiken CE, Fowden AL, Smith GC. Antenatal glucocorticoids prior to cesarean delivery at term. JAMA Pediatr 2014;168:507-8.

4 Holmes MC, Sangra M, French KL, et al. 11Betahydroxysteroid dehydrogenase type 2 protects the neonatal cerebellum from deleterious effects of glucocorticoids. Neuroscience 2006;137:865-73.

5 Wyrwoll CS, Holmes MC, Seckl JR. $11 \beta$-Hydroxysteroid dehydrogenases and the brain: from zero to hero, a decade of progress. Front Neuroendocrinol 2011;32:265-86.

6 Heine VM, Rowitch DH. Hedgehog signaling has a protective effect in glucocorticoidinduced mouse neonatal brain injury through an 11 betaHSD2-dependent mechanism. J Clin Invest 2009;119:267-77.

7 Crudo A, Suderman M, Moisiadis VG, et al. Glucocorticoid programming of the fetal male hippocampal epigenome. Endocrinology 2013;154:1168-80.

8 Stutchfield PR, Whitaker R, Gliddon AE, et al. Behavioural, educational and respiratory outcomes of antenatal betamethasone for term caesarean section (ASTECS trial). Arch Dis Child Fetal Neonatal Ed 2013;98:F195-F200.

9 Dalziel SR, Lim VK, Lambert A, et al. Antenatal exposure to betamethasone: psychological functioning and health related quality of life 31 years after inclusion in randomised controlled trial. BMJ 2005;331:665

10 Weikum ER, Knuesel MT, Ortlund EA, et al. Glucocorticoid receptor control of transcription: precision and plasticity via allostery. Nat Rev Mol Cell Biol 2017:18:159-74.

11 Braithwaite RS. A piece of my mind. EBM's six dangerous words. JAMA 2013;310:2149-50. 